\title{
Peertechz
}
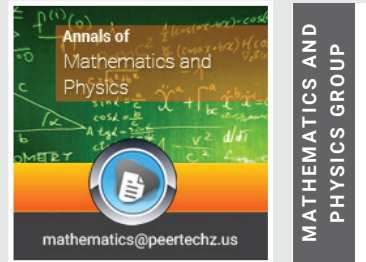

\section{Application of algebra to trisect an angle of 60 degree}

\section{Sivasubramanian ${ }^{1}$ and S Kalimuthu ${ }^{2 *}$}

'Department of Mathematics, Dr. Mahalingam College of Engineering and Technology, Pollachi,

Tamilnadu-642 003, India

Received: 17 July, 2020

Accepted: 16 April, 2021

Published: 19 April, 2021

*Corresponding authors: S Kalimuthu, SF 211 \& 212/4, Kanjampatti P.O, Pollachi Via, TamilNadu - 642 003, India, E-mail: nineteen4@gmail.com

Keywords: Algebra; Geometry; Angles; Trisection; Galois theory; Pierre wintzel and Lindemann

https://www.peertechzpublications.com

\section{Check for updates}

${ }^{2}$ SF 211 \& 212/4, Kanjampatti P.O, Pollachi Via, TamilNadu - 642 003, India

\section{Abstract}

Trisection of an angle, doubling the cube, squaring the circle, to draw a regular septagon and to deduce Euclid V from Euclid I to IV are the famous classical impossibilities. Recently, Sivasubramanian and Kalimuthu jointly and independently found several solutions for the parallel postulate problem. Their findings have been published in various peer reviewed international journals. In this work, by applying linear algebraic equations the authors have attempted and trisected 60 degree without using a protractor

\section{Introduction}

The origins of trisection of an angle began around $500 \mathrm{BC}$. Many many great mathematicians tried their best to solve this problem but miserably failed. Trisection of an angle and doubling the cube were proved impossible by Pierre Wintzel in 1837, although their impossibility was already known to Gauss in 1800. Squaring the circle problem was proved to be impossible by Lindemann in 1882 [1-7]. Both Wintzel and Lindemann applied the laws of Galois field theory and derived their results. It is to be noted that in quantum mechanics and super string theories the basics of abstract algebra particularly the laws of Lie groups are widely applied. It is a well known fact that the predictions of Einstein's special and general relativity theories have been experimentally established. But there are published experimental tests which challenge these theories. i.e. in some experiments these predictions do not hold [8-18].

Similarly, the authors findings also challenge abstract algebra. The authors never never and never question the consistent field of abstract algebra. But the authors firmly believe that the laws of abstract algebra can not fetter the angle trisection.

\section{Construction}

Construct an equilateral triangle $\mathrm{ABC}$ in Figure 1. On the extensions of $\mathrm{AB}$ and $\mathrm{AC}$, make $\mathrm{BD}=\mathrm{DE}=\mathrm{AB}$ and $\mathrm{AC}=\mathrm{CE}=\mathrm{EF}$ respectively. Join $\mathrm{B}$ and $\mathrm{F} \& \mathrm{D}$ and $\mathrm{F}$. Bisect $\mathrm{BD}$ at $\mathrm{G}$. Join $\mathrm{G}$ and

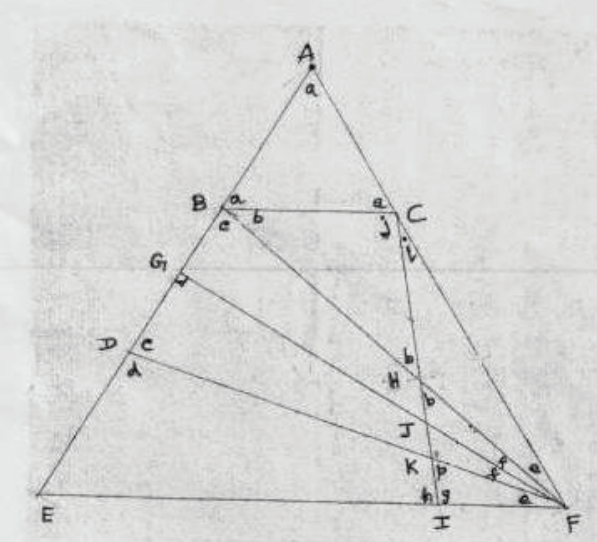

Figure 1: Construct an equilateral triangle.

F. With centre $\mathrm{C}$ radius $\mathrm{CB}$ describe an arc cutting $\mathrm{BF}$ at $\mathrm{H}$. Join $\mathrm{C}$ and $\mathrm{H}$ and produce it till it meets $\mathrm{EF}$ at I and GF at J.

In triangles $\mathrm{ABC}$ and $\mathrm{AEF}$ all the sides are equal and all the angles are equal. In triangle $\mathrm{BCH}$, since $\mathrm{BC}$ and $\mathrm{HC}$ are equal, the base angles are equal. By SAS correspondence triangles AGF and EGF are congruent. So, the angle at $\mathrm{G}$ is 90 degree. By SAS correspondence triangles BGF and DGF are congruent. So, angles GBF and GDF are equal, and angles BFG and DFG are equal. By SAS correspondence triangles $\mathrm{ABF}$ and EDF are congruent. So, angles AFB and EFD are equal, and angles $\mathrm{ABF}$ 
and EDF are equal.

Let the sum of the interior angles of all Euclidean triangles is equal to 180 degrees.

\section{Application of Algebra to Trisect an Angle of 60 Degree}

From the above constructions,

$b+c=j+i$

$a+b=d$

$2 e+2 f=a$

In triangle IFC,

Using (5) in RHS,

In triangles $\mathrm{BHC}$ and IHF,

Using (6) in RHS,

In triangle $\mathrm{CHF}$,

Equating (7) and (8),

In triangle CIF,

Assuming (1),

Adding the above two,

Using (6) in LHS,

$a+j=2 e+2 f+g$

$j=g$

$b+j=g+2 e+2 f$

$b=e+2 f$

$\mathrm{b}=\mathrm{e}+\mathrm{i}$

$\mathrm{i}=2 \mathrm{f}(9)$

$h=4 f+2 e$ by using

$2 b+j=2 c+2 f$

$2 b+j+h=6 f+2 e+2 c$

180 degree $+2 b=6 f+2 e+c$

Let us apply mathematical induction in (10).

Put $e=2 f$

So, (10) becomes,

In triangle $\mathrm{BCH}$

Using (11b) in (11a),

$2 b+d=10 f+c$
$2 b=180$ degree $-j$.

(11b)

180 degree $+d=10 f+c+j$

In triangle $\mathrm{BGF}, \mathrm{c}+\mathrm{f}=90$ degree.

Applying this in (12), 90 degree $+d=9 f+j$

From straight angles at $\mathrm{B}$ and $\mathrm{D}, \mathrm{d}=\mathrm{a}+\mathrm{b}$. Putting this in (13), 90 degree $+a+b=9 f+j$

Substituting (5) in (14),

Using (11) in (15)

90 degree $+2 e+2 f+b=9 f+j$

90 degree $+6 f+b=9 f+j$

i.e. $\quad 90$ degree $+b=3 f+j$

Using (12) in (16),

Applying (9) in (17),

$b+c=2 f+j$

$b+c=j+i$

So, if we put e $=2 \mathrm{f}$ in (10), we yield (3) and there is no contradiction. In other words equation (3) can be deduced by replacing e by $2 \mathrm{f}$ in equation (10). So, e $=2 \mathrm{f}$ is the acceptable solution.

Applying $\mathrm{e}=2 \mathrm{f}$ at angle $\mathrm{c}, 3 \mathrm{e}=60$ degrees. So, $\mathrm{e}$ is 20 degree .

\section{Discussion}

For trial measuring angle e, $2 \mathrm{f}$ and $\mathrm{i}$, we get that $\mathrm{e}=2 \mathrm{f}=\mathrm{i}=\mathbf{2 0}$ degree. Describing an arc with center $\mathrm{H}$ and radius $\mathrm{HC}$, it moves through F. So, e = 20 degree is consistent. In this work, we have not introduced or assumed any conjecture or hypothesis. Only we have applied one of the fundamental operations of number theory. ( i.e. addition ). So, beyond any doubt $e=2 f=20$ degree is consistent.

\section{Conclusion}

The authors attempts will open the further attempts which may explore new and fascinating results.

\section{Acknowledgement}

The authors are very grateful to the late professor Palaniappan Kaliappan, Department of Mathematics, Nallamuthu Gounder Mahalingam College, Pollachi, Tamilnadu-642001 India for introducing and encouraging the authors to work on this famous problem. The authors also whole heartedly thank Arutchelvar Padma Bhoosan Dr. N. Mahalingam Esquired Chairman, Dr. Mahalingam College of Engineering and Technology, Pollachi, Tamilnadu-642003, India for his kind encouragement and magnanimous monetary support for the preparation of this work. 


\section{References}

1. Eves H (1976) An Introduction to the history of Mathematics, Holt, Rhinehart and Winston, New York. Link: https://bit.ly/3tsHPp6

2. Dunham W (1990) Journey through Genius John Wiley and sons. Link: https://bit.ly/3gndZP4

3. Stewart I, Theory G (1991) Chapman and Hall, London.

4. Herstein IN (1964) Topics in Algebra, Blasdell, New York.

5. Jones A, Morris AS, Person KR (1991) Abstract Algebra and Famous Impossibilities, Springer-Verlag, New York. Link: https://bit.ly/32iZ3cy

6. Courant R, Robbins H (1941) What is mathematics?, Oxford University Press, New York.

7. Charles RH (1978) Field Theory and its Classical Problems, Carus Mathematical Monographs, No. 19, Mathematical Association of America.

8. Sivasubramanian M, Kalimuthu S (2008) On the New Branch of Mathematical Science. Journal of Mathematics and Statistics 4: 122-123. Link: https://bit.ly/3aenOuW

9. Sivasubramanian M, Senthilkumar L, Raghul Kumar K, Kalimuthu S (2008) On the New Branch of Mathematical Science - Part 2. Journal of Mathematics and Statistics 4: 148-149. Link: https://bit.ly/3silN64
10. Sivasubramanian M (2009) Application of Sivasubramanian Kalimuthu Hypothesis to Triangles. Journal of Mathematics and Statistics 5: 90-92. Link: https://bit.ly/3wUxnJn

11. Sivasubramanian M (2009) A phenomenon in geometric Analysis. Indian Journal of Science and Technology 2: 23 - 24.

12. Sivasubramanian M (2009) Application of Algebra to Geometry. Indian Journa of Science and Technology 2: 1-2.

13. Sivasubramanian M (2009) On the parallel postulate. Researcher, Marsland press 1: 58-61.

14. Sivasubramanian M, Kalimuthu S (2009) A Computer Application in Mathematics. Computers and Mathematics with Applications, Elsevier Publications, The Netherlands.

15. Kalimuthu S (2009) "Pretty Algebra", Nature and science, Marshland Press, New York 7: 86-89. Link: https://bit.ly/3wUzqNz

16. Kalimuthu S (2009) "Beautiful geometry", Nature and science, Marshland Press, New York 7: 88-89.

17. Kalimuthu S (2009) For the origin of quantum geometry (Submitted to Applied Mathematics letters)

18. Kalimuthu $S$ (2009) The parallel postulate-The return of the roaring line. Indian Journal of Science and Technology 2: 6-22. Link: https://bit.ly/32nfc0G
Discover a bigger Impact and Visibility of your article publication with

Peertechz Publications

Copyright: (C) 2021 Sivasubramanian M, et al. This is an open-access article distributed under the terms of the Creative Commons Attribution License, which permits unrestricted use, distribution, and reproduction in any medium, provided the original author and source are credited. 